\title{
Pulmonary Toxicity Following an Intratracheal Instillation of Nickel Oxide Nanoparticle Agglomerates
}

\author{
Yasuo Morimoto ${ }^{1}$, Masami Hirohashi ${ }^{1}$, Akira Ogami ${ }^{1}$, Takako Oyabu${ }^{1}$, Toshihiko Myojo ${ }^{1}$, \\ Masayoshi HashibA ${ }^{1}$, Yohei Mizuguchi ${ }^{1}$, Tatsunori Kambara ${ }^{1}$, Byeong Woo LeE ${ }^{1}$, \\ Etsushi KurODA ${ }^{2}$ and Isamu TANAKA ${ }^{1}$ \\ ${ }^{1}$ Institute of Industrial Ecological Sciences and ${ }^{2}$ Department of Immunology and Parasitology, School of Medicine, \\ University of Occupational and Environmental Health, Japan
}

\begin{abstract}
Pulmonary Toxicity Following an Intratracheal Instillation of Nickel Oxide Nanoparticle Agglomerates: Yasuo Mовıмото, et al. Institute of Industrial Ecological Sciences, University of Occupational and Environmental Health, JapanObjective: We examined the pulmonary toxicity of nickel oxide nanoparticle agglomerates in the rat lung following an intratracheal instillation. Methods: The weighted average surface primary diameter of nickel oxide nanoparticles was $8.41 \mathrm{~nm}$, and the count median diameter of nickel oxide nanoparticle agglomerates suspended in saline was $1.34 \mu \mathrm{m}$. Male Wistar rats were exposed to $1 \mathrm{mg}(3.3 \mathrm{mg} / \mathrm{kg})$ of nickel oxide nanoparticles intratracheally. The control group received intratracheal instillation of saline. Rats were dissected 3 days, $1 \mathrm{wk}, 1 \mathrm{mo}, 3 \mathrm{mo}$, and 6 mo after the instillation. Cytokine-induced neutrophil chemoattractant (CINC)$2 a \beta$ in the lung tissue was determined by quantitative measurement of protein by ELISA. Results: The total cell count in bronchoalveolar lavage fluid (BALF) was increased persistently from 3 days to $6 \mathrm{mo}$. The neutrophil counts in BALF were also increased at 3 days, $1 \mathrm{wk}, 3 \mathrm{mo}$, and $6 \mathrm{mo}$. In the lung tissue, infiltration of mainly neutrophils and alveolar macrophages was observed in alveoli from 3 days to 6 mo. The CINC-2a $\beta$ concentration was elevated from 3 days to $6 \mathrm{mo}$ in the lung tissue. Conclusions: These results showed that micron-sized nickel oxide nanoparticle agglomerates also induced a persistent inflammatory response.

(J Occup Health 2011; 53: 293-295)
\end{abstract}

Received Feb 4, 2011; Accepted Apr 14, 2011

Published online in J-STAGE May 18, 2011

Correspondence to: Y. Morimoto, Department of Occupational Pneumology, Institute of Industrial Ecological Sciences, University of Occupational and Environmental Health, Japan, Yahatanishiku, Iseigaoka 1-1, Kitakyushu, Fukuoka 807-8555, Japan

(e-mail: yasuom@med.uoeh-u.ac.jp)
Key words: Agglomeration, Intratracheal instillation, Nanoparticles, Nickel oxide, Rat

Manufactured nanomaterials have been demanded according to the development of the nanotechnology and their structure is such that at least one of 3 dimensions is about $1-100 \mathrm{~nm}{ }^{1)}$. One of these nanomaterials, nickel oxide, has been used for ceramic, as a catalyst, and for storage battery, and has also been reported to induce inflammatory responses in the lung in vivo studies ${ }^{2)}$. However, detailed information on the lung toxicity of nickel oxide nanoparticles is needed because the differences in the physicochemical properties of the materials reflect the pulmonary response ${ }^{2}$. Therefore, it is very important to characterize nickel oxide nanomaterials to estimate their harmful effects.

Therefore, we examined pulmonary inflammation in the rat lung following an intratracheal instillation of wellcharacterized nickel oxide nanoparticles.

\section{Materials and Methods}

Animals

Male Wistar rats were purchased from Kyudo Co., Ltd. (Kumamoto, Japan). All procedures and animal handling were performed according to the guidelines described in the Japanese Guide for the Care and Use of Laboratory Animals as approved by the Animal Care and Use Committee, University of Occupational and Environmental Health, Japan.

\section{Characterization of nickel oxide nanoparticles}

A nickel oxide nanoparticle sample (20 nm nominal primary diameter, $99.8 \%$ purity) was purchased from Nanostructured and Amorphous Materials, Inc. The Brunauer-Emmett-Teller (BET) specific surface area of the measured sample was $104.6 \mathrm{~m}^{2} / \mathrm{g}$, and the weighted average surface primary diameter (Sauter diameter) was 


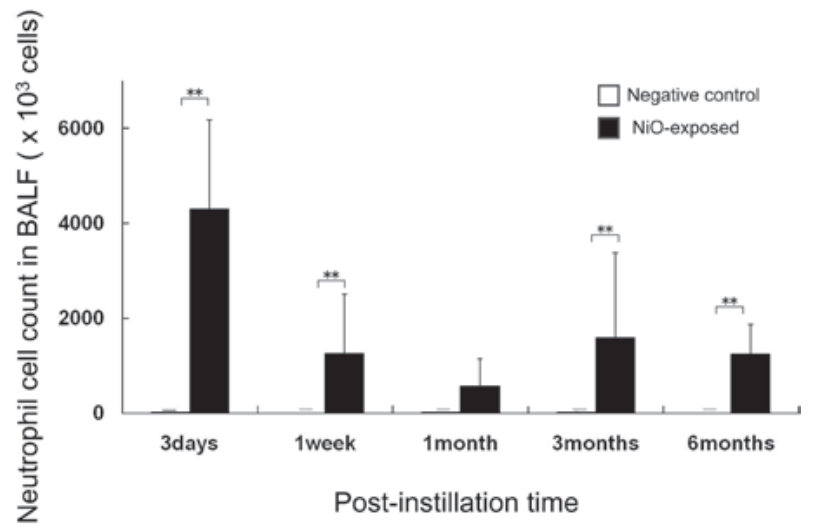

Fig. 1. Neutrophil counts in BALF exposed to nickel oxide nanoparticles. Each column and bar represents the mean $\pm \mathrm{SD}$ of five rats. Double asterisks indicate significant differences at $p<0.01$ versus each control group. Nickel oxide nanoparticle agglomerates induced persistent neutrophil inflammation in the rat lung.

$8.41 \mathrm{~nm}$. The nickel oxide nanoparticles were suspended with saline and adjusted to $2.5 \mathrm{mg} / \mathrm{ml}$. The nickel oxide nanoparticles suspension was dispersed with a bath type of ultrasonication for $30 \mathrm{~min}$ and used in the intratracheal instillation study.

\section{Intratracheal instillation}

Nickel oxide nanoparticles suspension was mixed with $0.4 \mathrm{ml}$ saline, and $1 \mathrm{mg}(3.3 \mathrm{mg} / \mathrm{kg})$ nickel oxide was intratracheally instilled once to male Wistar rats $(9 \mathrm{wk}$ old). The negative control groups received $0.4 \mathrm{~m} l$ of saline. Animals were dissected at 3 days, $1 \mathrm{wk}, 1 \mathrm{mo}, 3$ mo, and 6 mo after the instillation. Each group of 10 animals was divided into 2 subgroups of 5 animals for lung tissue analysis. One lung of the first subgroup was homogenized to extract protein. The second subgroup provided bronchoalveolar lavage fluid, which was collected using physiological saline that was poured through a cannula inserted in the respiratory tract into right lung. Three to $10 \mathrm{~m} l$ of physiological saline was infused each time, and up to a total of $50 \mathrm{~m} l$ of lavage fluid was collected by free-fall.

\section{Chemokine measurement of lung tissue and BALF}

The lung tissue was homogenized with tissue protein extraction reagent (T-PER), Halt protease inhibitor, and Cocktail Kit, and then centrifuged. The total protein concentration was adjusted to a final concentration of 500 $\mu \mathrm{g} / \mathrm{m} l$ for cytokine-induced neutrophil chemoattractant$2 \alpha \beta$ (CINC-2 $\alpha \beta$; R\&D Systems; Cat. \# RCN200).

\section{Results}

Characterization of nickel oxide nanoparticles

The count median cumulative 10\%, 50\% and $90 \%$

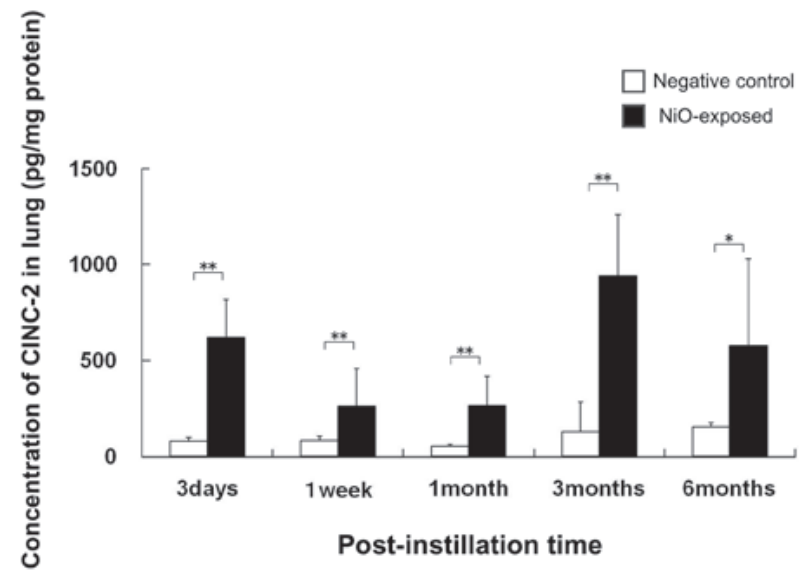

Fig. 2. CINC-2 $\alpha \beta$ concentration in lung tissue exposed to nickel oxide nanoparticles. Each column and bar represents the mean $\pm \mathrm{SD}$ of five rats. An asterisk is used to indicate significant differences at $p<0.05$ versus each control group; double asterisks indicate significant differences at $p<0.01$ versus each control group. Nickel oxide nanoparticle agglomerates induced a persistent concentration of CINC- $2 \alpha \beta$ in the rat lung.

diameters of the nickel oxide nanoparticle agglomerates suspended in saline for intratracheal instillation were 0.48 $\mu \mathrm{m}, 1.34 \mu \mathrm{m}$ and $8.69 \mu \mathrm{m}$, as determined by a Microtrac FRA (Microtrac, Montgomeryville, PA, USA) utilizing laser diffraction and light scattering as a measurement method. The proportion of particles with a diameter larger than $1 \mu \mathrm{m}$ was approximately $60 \%$. These data suggested that the particles were micron-sized agglomerates.

\section{Total and neutrophil count in BALF}

The total cell count in BALF was persistently increased in the nickel oxide-exposed groups from 3 days to 6 mo after the instillation. Compared with the negative control groups, the neutrophil counts in BALF continued increasing in the nickel oxide-exposed groups at 3 days, 1 wk, 3 mo and 6 mo (Fig. 1).

\section{CINC- $2 \alpha \beta$ concentration in lungs}

The CINC- $2 \alpha \beta$ concentration in lung tissue was significantly and persistently elevated from 3 days to 6 mo in the $1 \mathrm{mg}$ nickel oxide-exposed group as compared with the negative control group (Fig. 2). The peak of the CINC$2 \alpha \beta$ concentration was observed at 3 mo.

\section{Histopathological changes in lungs}

In the nickel oxide-exposed group, the infiltration of polymorphonuclear cells and foamy macrophages was persistently observed in the alveolar space throughout the observation period, and alveolar proteinosis was remarkable from 3 mo after instillation. 


\section{Discussion}

In our previous studies, nano-sized nickel oxide nanoparticle agglomerates induced pulmonary inflammation in the rat lung in intratracheal instillation studies $^{3,4)}$. The agglomerated size of the nickel oxide nanoparticles was at the micron level in this study, and we found that micron-sized nickel oxide nanoparticles induced persistent pulmonary inflammation in the intratracheal instillation study. On the other hand, nickel oxide with a micron-sized diameter did not induce pulmonary inflammation in the rat lung in an intratracheal instillation study ${ }^{5}$. Therefore, nickel oxide nanoparticles might also induce a pulmonary response such as titanium dioxide in spite of the size of agglomerates ${ }^{6}$.

We examined the relationship of infiltration of neutrophils and CINC in this study, and nickel oxide nanoparticle agglomerates induced a persistent increase in the concentration of CINC- $2 \alpha \beta$ in lung tissue. Diesel particles $^{7)}$ and crystalline silica ${ }^{3)}$ with inflammatory potentials have been reported to induce a persistent increase in CINC-1 or CINC-2 expression in the lung in intratracheal instillation studies. In previous intratracheal instillation studies, pulmonary inflammation was accompanied by expression of CINCs, and the pathological and CINC data in the present study are consistent with the results of previous studies.

\section{Conclusion}

We examined the pulmonary toxicity of nickel oxide nanoparticle agglomerates following an intratracheal instillation and found that infiltration of neutrophils and alveolar macrophages including foamy macrophages and upregulation of CINC families were observed persistently in rat lungs during the observation periods. These data suggested that micron-sized nickel oxide nanoparticle agglomerates have inflammatory potentials that lead to irreversible chronic lesions such as fibrosis or cancer.

Acknowledgments: This research was partially funded by a Grant-in-Aid for Scientific Research (C; 20590939).

\section{References}

1) ISO Nanotechnologies-Terminology and definitions for nanoparticles. 2008; ISO-TS 27687

2) Morimoto Y, Kobayashi N, Shinohara N, Myojo T, Tanaka I, Nakanishi J. Hazard assessments of manufactured nanomaterials. J Occup Health 2010; 52: 325-34.

3) Nishi K, Morimoto Y, Ogami A, et al. Expression of cytokine-induced neutrophil chemoattractant in rat lungs by intratracheal instillation of nickel oxide nanoparticles. Inhal Toxicol 2009; 21: 1030-9.

4) Morimoto Y, Ogami A, Todoroki M, et al. Expression of inflammation-related cytokines following intratracheal instillation of nickel oxide nanoparticles. Nanotoxicology 2010; 4: 161-76.

5) Ogami A, Morimoto Y, Myojo T, et al. Pathological features of different sizes of nickel oxide following intratracheal instillation in rats. Inhal Toxicol 2009; 21 : 812-8.

6) Kobayashi N, Naya M, Endoh S, Maru J, Yamamoto K, Nakanishi J. Comparative pulmonary toxicity study of nano- $\mathrm{TiO}_{2}$ particles of different sizes and agglomerations in rats : Different short- and long-term post-instillation results. Toxicol 2009; 264: 110-8.

7) Yokota S, Seki T, Furuya M, Ohara N. Acute functional enhancement of circulatory neutrophils after intratracheal instillation with diesel exhaust particles in rats. Inhal Toxicol 2005; 17: 671-9. 